Springer Link

- Research Article

- Published: 13 May 2016

\title{
CFD evaluation of pressure drop across a 3-D filter housing for industrial gas turbine plants
}

- Fidelis I. Abam,

- Samuel O. Effiom \&

- Olayinka S. Ohunakin

Frontiers in Energy volume 10, pages192-202(2016) Cite this article

- 230 Accesses

- 4 Citations

- Metricsdetails

\section{Abstract}

This paper investigated the flow distribution and total pressure drop across a designed 3-D filter housing integrated with a 3-stage filtration system using computational fluid dynamics (CFD). The filter housing model was proposed for a heavy-duty industrial gas turbine plant operating at an average ambient temperature of $20^{\circ} \mathrm{C}$. The pressure drops across the classes of filters were 652.8 $\mathrm{Pa}$, 2692.2 Pa, 887.8 Pa, 776.2 Pa and 2304.2 Pa for I-GB, GB-GA, GA-FA, FA$\mathrm{HA}$, and $\mathrm{HA}-\mathrm{O}$, respectively. The results obtained indicated an acceptable total pressure drop of $7.2 \%$ for the entire filter housing before filter clean-up. Although the CFD simulation result shows that small outlet flow velocity and transonic flows exist at the outlet of the filter housing, the designed filter housing was proved compatible with the studied GT, for inlet flow conditions between $600 \leqslant W_{\text {air }} \leqslant 610 \mathrm{~kg} / \mathrm{s}$ and $60 \leqslant V_{\text {air }} \leqslant 70 \mathrm{~m} / \mathrm{s}$ for the air flow rate and velocity, respectively. Furthermore, the designed filter housing could be adopted for the studied GT and locations of Usan and Maiduguri in Nigeria, and other locations with similar environmental conditions. 
This is a preview of subscription content, access via your institution.

\section{References}

1. 1.

Shweta N, Darshana B. Geometry optimization of filter house components fitted at compressor entry. International Journal Science and Research, 2014, 3(9): 905-909

\section{Google Scholar}

2. 2.

Effiom S O, Abam F I, Ohunakin O S. Performance modeling of industrial gas turbines with inlet air filtration system. Case study in Thermal Engineering, 2015, 5: 160-167

\section{Article Google Scholar}

3. 3 .

Wilcox M, Baldwin R, Garcia-Hernandez A, Brun K. Guideline for gas turbine inlet air filtration systems. In: Proceedings of the Gas Machinery Research Council. 2010. 2014-01-21, https://www.gmrc. org/documents/guidelineforgasturbineinletairfiltrationsystems.pdf

\section{Google Scholar}

4. 4 .

Chopade M R, Valavade A P, Barhatte S H. Performance evaluation of air filters by design optimization. International Journal of Advances in Engineering and Technology, 2012, 3(1): 68-70 


\section{Google Scholar}

5. 5 .

Manikantan R, Gunasekaran E J. Modeling and analyzing of air filter in airintake system in automobileengine. Advances in Mechanical Engineering, 2013, 1-7; Article ID 654396

\section{Google Scholar}

6. 6 .

Hosseinzadah S, Gorji-Bandpy M, Rad G J, Keshavarz M. Experimental and numerical study of impact of air filter holes masking on altitude at heavy-duty diesel engine. Modern Mechanical Engineering, 2012, 2(04): 157-166

\section{Article Google Scholar}

7. 7.

Khashayar K, Karimian S M H, Varmaziar M, Sarjami S. Investigation of flow pattern and pressure loss of a V94.2.5 gas turbine air intake system using 3D numerical modeling. 2014-12-22, http://www.wseas.us/elibrary/conferences/2010/Cambridge/CFH/ CFH-43.pdf

8. 8.

Feng Z, Long Z, Chen Q. Assessment of various CFD models for predicting airflow and pressure drop through pleated filter system. Building and Environment, 2014, 75: 132-141

\section{Article Google Scholar}


9. 9.

Fan J, Shah L J, Furbo S. Flow distribution in a solar collector panel with horizontally inclined absorber strips. Solar Energy, 2007, 81 (12): 15011511

\section{Article Google Scholar}

10.10 .

Fan J, Furbo S. Buoyancy effects on thermal behaviour of a flatplate solar collector. Journal of Solar Energy Engineering, 2008, 130: 1-12, 021010.

\section{Article Google Scholar}

11.11.

Fridolin K. CFD for air induction systems with open foam. Dissertation for the Master's Degree. Gothenburg: Chalmers University of Technology, 2012

\section{Google Scholar}

12.12.

Gourdain N. Application of CFD to turbomachine based systems. 201412-15, http://www.cerfacs.fr/ cfdbib/repository/TR_CFD_09_131.pdf

\subsection{3.}

Yerram R, Prasad N, Malathkar P R, Halbe V, Murthy S D. Optimization of intake system and filter of an automobile using CFD analysis. 2014-1215 , 
https://www.researchgate.net/publication/268340757_Optimization_of_I ntake_System_and_Filter_of_an_Automobile_using_CFD_analysis

14.14.

ANSYS Inc. ANSYS CFX 14.0 User's Guide. SAS IP Inc. 2012

15.15.

ANSYS Inc. ANSYS ICEM CFD 14.0 User Manual. SAS IP Inc. 2012

16.16 .

Theokilis N, Pachidis P. TUBOMATCH Scheme User Manual. Department of Power and Propulsion, Cranfield University, UK, 2014

\section{Google Scholar}

17.17 .

Climatemps. Climate,average weather of Nigeria 2014. 2014-05-25, http://www.nigeria.climatemps.com

18.18.

Shih T H, Liou W W, Shabbir A, Yang Z, Zhu J. A new $k-\varepsilon$ eddy viscosity model for high reynolds number turbulent flows. Computers \& Fluids, 1995, 24(3): 227-238

\section{Article MATH Google Scholar}




\section{Author information}

Affiliations

1. Department of Mechanical Engineering, Michael Okpara University of Agriculture, Umudike, Umuahia, 02155, Nigeria

Fidelis I. Abam

2. Department of Mechanical Engineering, Cross River University of Technology, Calabar, 540252, Nigeria

Samuel O. Effiom

3. Department of Mechanical Engneering, Covenant Universiy, Ota, 11001, Nigeria

Olayinka S. Ohunakin

Abam, F.I., Effiom, S.O. \& Ohunakin, O.S. CFD evaluation of pressure drop across a 3-D filter housing for industrial gas turbine plants. Front.

Energy 10, 192-202 (2016). https://doi.org/10.1007/s11708-016-0406-x

Download citation

- Received15 August 2015

- Accepted09 November 2015

- Published13 May 2016

- Issue DateJune 2016

- DOlhttps://doi.org/10.1007/s11708-016-0406-x

Keywords

- computational fluid dynamics (CFD)

- pressure drop

- flow distribution

- filter housing

- gas turbine

Over 10 million scientific documents at your fingertips

Switch Edition 
- Academic Edition

- Corporate Edition

- $\underline{\text { Home }}$

- Impressum

- Legal information

- $\quad$ Privacy statement

- California Privacy Statement

- $\quad$ How we use cookies

- Manage cookies/Do not sell my data

- Accessibility

- $\quad$ Contact us

Not logged in - 165.73.223.243

Not affiliated 


\section{Springer Nature}

(C) 2021 Springer Nature Switzerland AG. Part of Springer Nature. 